\title{
Near infrared spectroscopy: rethinking the analysis of sugarcane factory streams
}

\author{
S. Walford \\ Sugar Milling Research Institute NPC, c/o University of KwaZulu-Natal, Durban, 4041, South Africa. E-mail: swalford@smri.org
}

Near infrared spectroscopy (NIRS) for the simultaneous analysis of brix, pol, sucrose, glucose, fructose and ash in sugar factory processing streams has many advantages. The most important are the almost immediate reporting of results which can be used for process control, and the ability to analyse many samples over a short period for troubleshooting purposes. The Sugar Milling Research Institute NPC has developed and refined near infrared transmission spectroscopy models for the prediction of these analytes in sugarcane processing streams. The uptake of the technology in the sugar factory laboratories has proved challenging, requiring improved analyte prediction ranges, understanding the transfer of instrument results into factory information management systems and the introduction of a simplified sample dilution procedure. The NIRS technology has allowed for the development of toolkits for routine factory scale sucrose inversion studies and measurement of losses across centrifuges, previously difficult to implement.

\section{Introduction}

The Sugar Milling Research Institute NPC (SMRI) has been investigating the potential of using near infrared spectroscopy (NIRS) as an alternative analysis technique for the simultaneous analysis of brix, pol, sucrose, glucose, fructose and ash in sugar factory processing streams for more than 20 years. ${ }^{1-9}$ Sample presentation methods and associated equation development that were investigated included reflectance, transflectance and transmission techniques. The latter was adopted as the precision and accuracy was comparable to the existing methods. An added advantage was that the majority of factory samples are diluted for conventional analysis (brix and polarimetry), making technology transfer at the analyst level more simple.

The South African sugar industry is unique in that all factory balances and grower payments are based on sucrose and not pol (the apparent sucrose measured using polarimetry). Pol does not predict sucrose well in low purity products such as molasses where the sucrose content is typically $34 \%$ and pol content $27 \%$. Factory laboratories measure pol as sucrose analysis is difficult in a factory laboratory environment. Sucrose in the factory is calculated retrospectively based on weekly pol/sucrose factors provided by the SMRI Transmittance NIRS therefore offers the industry, not only a rapid analysis method but analytes that are not measured in the factory environment such as sucrose, glucose and fructose. This will also allow the factory personnel to control the unit operations directly, based on the product they are producing (sucrose) and not an apparent sucrose which is not a true value. This paper highlights the challenges in the technology transfer to an industry that has been fixated on methods that are over one hundred years old.

\section{Equation development and problems?}

In considering near infrared transmission spectroscopy technology as an alternative analysis method within a sugarcane factory environment, a number of constraints were identified. The current payment systems within the industry required prediction equations for the front end of the factory

\section{Correspondence}

S.Walford (swalford@smri.org)

doi: $10.1255 /$ nir2017.129

Citation: S. Walford, "Near infrared spectroscopy: rethinking the analysis of sugarcane factory streams", in Proc. $18^{\text {th }}$ Int. Conf. Near Infrared Spectrosc., Ed by S.B. Engelsen, K.M. Sørensen and F. van den Berg. IM Publications Open, Chichester, pp. 129-134 (2019). https://doi.org/10.1255/nir2017.129

\section{(c) 2019 The Author}

This licence permits you to use, share, copy and redistribute the paper in any medium or any format provided that a full citation to the original paper is given, the use is not for commercial purposes and the paper is not changed in any way. 
(juices) and the final waste product (molasses) that gave results that did not financially prejudice any one factory or grower. For factory control, the intermediate products equations (approximately 13 factory process streams) were not required to have as tight a precision. Analysis of raw sugar by NIRS was postponed at this stage. The industry required global equations that could operate at all 14 sugar factories with samples that were geographically sourced from rainfed and irrigated regions with different cane varieties and age of cane and could be processed in one of two different extraction process (diffusion vs milling) that resulted in different input streams. The alternative method was also expected to be robust and to provide equivalent results.

Prediction equations were developed using a $1 \mathrm{~mm}$ path length flow-through cell. Juices were analysed directly whilst all other samples were diluted to a standard mass/mass dilution. The technology was successfully implemented in the SMRI ISO 17025 accredited laboratory from the beginning of the $2009 / 10$ crushing season and was used for three years for the analysis and reporting of juice and final molasses samples. A combination of two near infrared transmission spectroscopy instruments and three cells were used in the successful implementation. Based on the success at the SMRI, an industry decision was made to transfer the technology to the factory environment. Fourteen factories purchased transmission instruments and transmission cells and the SMRI prediction equations were installed on the instruments. Within a few weeks it was apparent that ongoing, random large differences were noted between conventional and NIRS predictions making factory control difficult and the technology was withdrawn from the factories.

Investigation showed that the non-transferability of predictions between the factory and SMRI instruments was caused by the "OS" optical glass transmission cells" that were being used. The optical glass cells contained differing amounts of low concentrations of water which interfered with the transmission scan. SMRI had previously checked three "OS" cells which had been purchased over a one-year period and found to give acceptable results. Enquiries with the manufacturer showed that, although purchased over an extended period, all three cells were from one production batch. Matched "QS" quartz cells were investigated, but also showed trace amounts of water in the quartz, albeit much lower. In discussion with the supplier, it became apparent that the

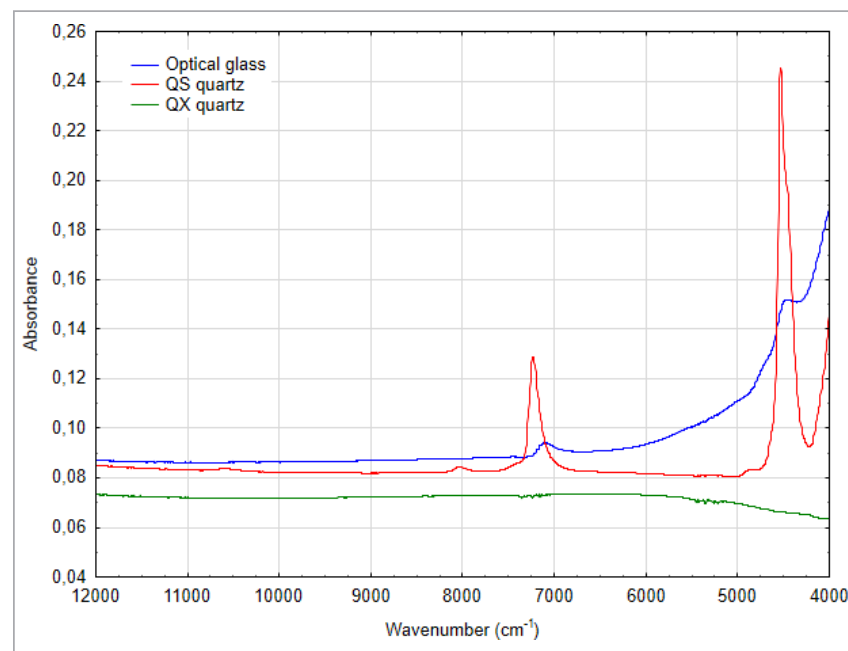

Figure 1. Absorbance spectra of transmission cells manufactured from "OS" optical glass (blue), "QS" quartz (red) and "QX" quartz, showing presence and absence of water within the cell material.

cells were "matched" to within 1\% in path length and that no check was performed as to the variance in cell absorption across the NIR wavelength range. The cell manufacturer suggested "QX" quartz cells and these proved acceptable and were used for all subsequent prediction development and routine analysis (Figure 1).

To ensure inclusion of as wide a variance as possible within the prediction equations, thirteen factory instruments were loaned to the SMRI for a five-month period. During this time scans of calibration samples were taken on all fifteen instruments and used as the basis of the equation development. Approximately 1000 factory samples were analysed resulting in more than 25,000 scans of factory products at this point in 2014. These were divided into a calibration/validation sets and independent test sets (Figure 2, Figure 3). Care was taken to ensure that no duplicate samples were present in both sets. Normal maintenance has continued since. The current state of the equations is shown for some of the analytes in Table 1.

Previously a peristaltic pump and associated tubing had been used to introduce the sample into the transmission cell. This had proved problematic in that sample analysis times were extended and dilute sugars solutions were often left in the tubing which fermented. To overcome this, a simple plastic-syringe-based introduction system incorporating Luer connectors was introduced with short connecting tubes and a flushing scheme to replace sugar solutions within the tubes. 


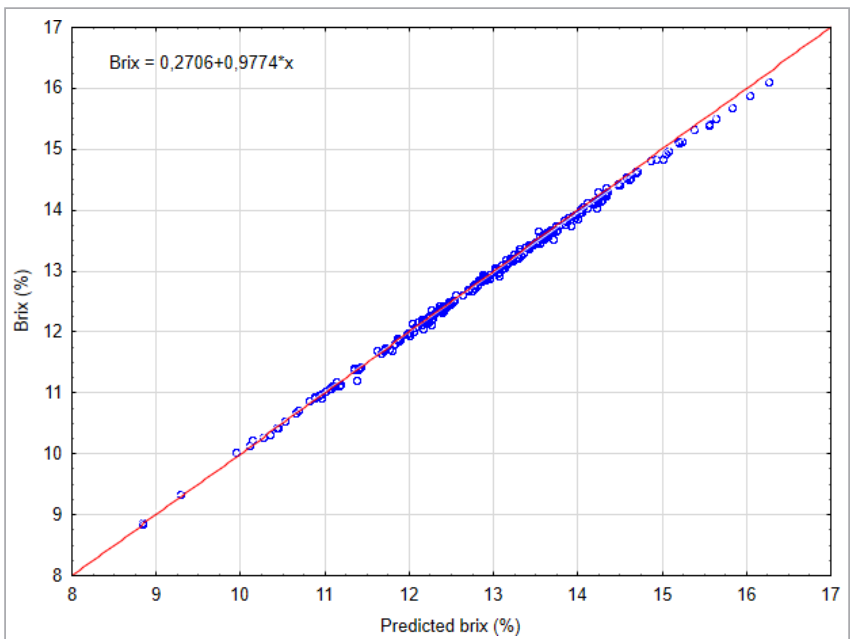

Figure 2. Predicted vs conventional juice brix for an independent set of 3563 samples collected over a ninemonth crushing season using a PLS model. Note that the prediction equation range for the season was 9.5-15.5\% brix with samples outside of this range being predicted well. The red line indicates the equivalence value.

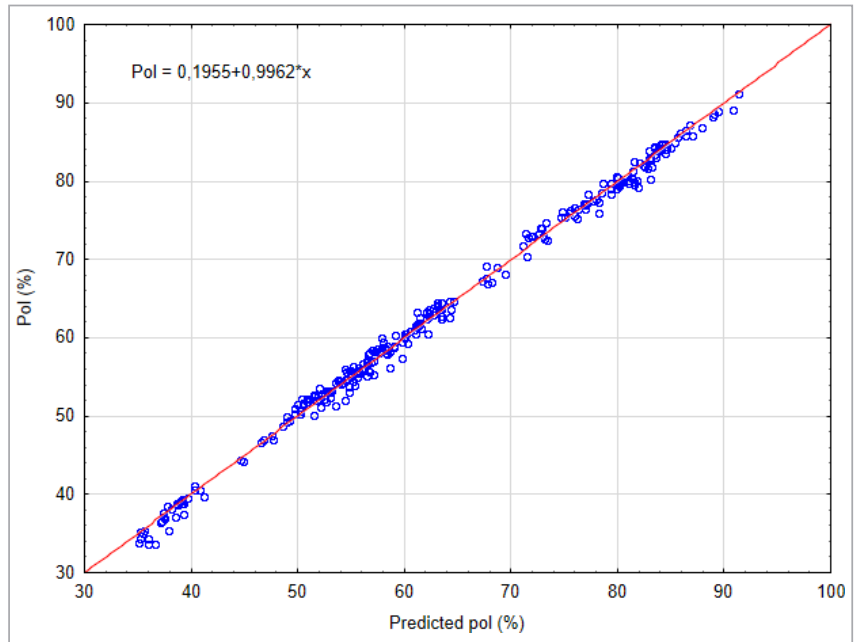

Figure 3. Predicted vs conventional intermediate pol for an independent sample set of 269 samples collected over three crushing seasons using a PLS model. The red line indicates the equivalence value.

Factory laboratories again started using the technology from the start of the 2014/15 season. Both the juice and molasses equations have been used successfully for the routine analysis of brix, pol, sucrose, glucose, fructose, ash and dry solids. However, there has been a reluctance to embrace the technology for routine analysis within the industry using the intermediate stream prediction equations.

\begin{tabular}{|c|c|c|c|c|c|}
\hline \multicolumn{2}{|c|}{ 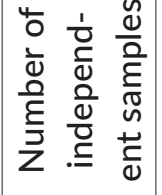 } & 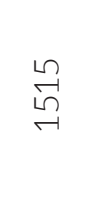 & 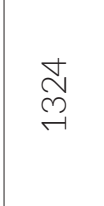 & $\underset{\stackrel{\sim}{\sim}}{\stackrel{ \pm}{\sim}}$ & $\begin{array}{l}0 \\
\infty \\
b \\
\rightarrow-1\end{array}$ \\
\hline \multicolumn{2}{|r|}{ 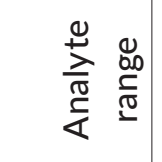 } & 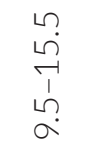 & 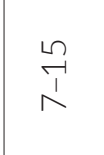 & $\begin{array}{l}\overrightarrow{7} \\
\vec{b}\end{array}$ & $\begin{array}{l}\text { O } \\
\text { I } \\
\text { } \\
\text { r }\end{array}$ \\
\hline \multirow{3}{*}{ 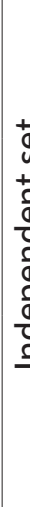 } & $\frac{n}{\frac{n}{\omega}}$ & $\begin{array}{l}m \\
8 \\
8 \\
\dot{0} \\
1\end{array}$ & $\begin{array}{l}\check{8} \\
\text { O. }\end{array}$ & $\begin{array}{l}-1 \\
8 \\
0 \\
\\
1\end{array}$ & స̃ \\
\hline & 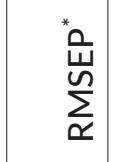 & $\begin{array}{l}m \\
\text { } \\
O \\
0\end{array}$ & $\begin{array}{l}\stackrel{\bigcirc}{M} \\
\rightarrow \\
0\end{array}$ & $\begin{array}{c}\stackrel{N}{N} \\
\stackrel{+}{0}\end{array}$ & $\begin{array}{l}\stackrel{\sim}{\sim} \\
\sim\end{array}$ \\
\hline & 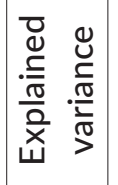 & $\begin{array}{l}\stackrel{\circ}{\alpha} \\
\stackrel{\alpha}{\alpha}\end{array}$ & 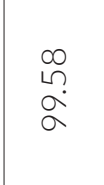 & $\begin{array}{l}\infty \\
\text { m. } \\
\alpha \\
\alpha\end{array}$ & $\begin{array}{l}m \\
\text { bo } \\
\stackrel{\alpha}{\alpha}\end{array}$ \\
\hline \multirow{2}{*}{$\begin{array}{l}\frac{1}{c} \\
v \\
\Delta \\
a \\
r\end{array}$} & 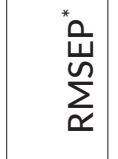 & $\begin{array}{l}\stackrel{0}{ } \\
\stackrel{0}{0} \\
0\end{array}$ & $\begin{array}{l}m \\
\stackrel{1}{0} \\
0\end{array}$ & $\stackrel{\substack{f \\
\hdashline}}{\circ}$ & 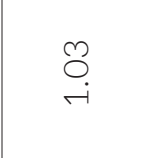 \\
\hline & 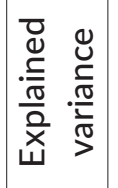 & $\begin{array}{l}\frac{n}{\alpha} \\
\alpha \\
\alpha\end{array}$ & $\begin{array}{l}\vec{b} \\
\stackrel{a}{a} \\
\alpha\end{array}$ & 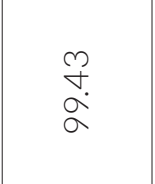 & $\begin{array}{l}\mathcal{N} \\
\omega^{2} \\
\infty\end{array}$ \\
\hline & 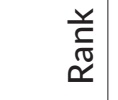 & 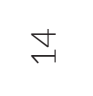 & $\stackrel{ન}{二}$ & $\stackrel{m}{\rightarrow}$ & $\underset{\sim}{\sim}$ \\
\hline & 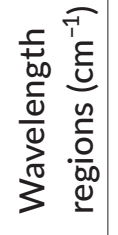 & 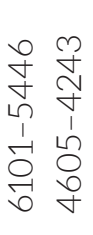 & 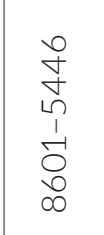 & 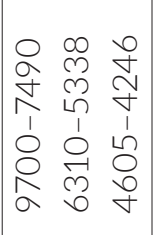 & 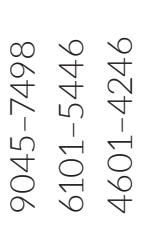 \\
\hline & 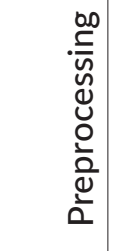 & 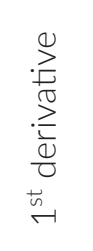 & 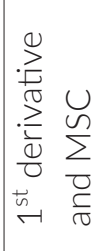 & 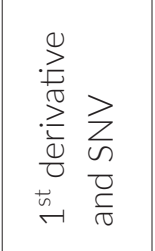 & 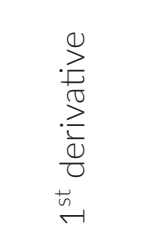 \\
\hline & $\begin{array}{l}\frac{\pi}{2} \\
\frac{\pi}{\pi} \\
\frac{\pi}{4}\end{array}$ & 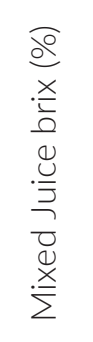 & 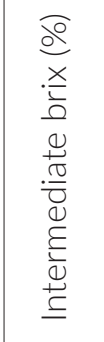 & 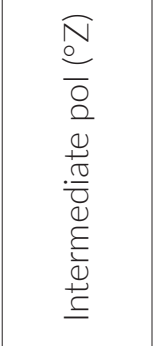 & 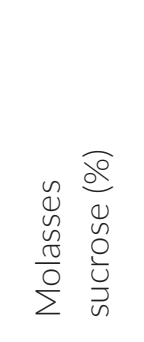 \\
\hline
\end{tabular}




\section{Hurdles to acceptance}

Investigation into the resistance factors hampering the adoption of the new technology showed that there were two major hurdles to acceptance. The first was that factory staff regarded the conventional results as the absolute truth. In comparing conventional and near infrared transmission spectroscopy results for the two analytes they used (brix and pol), the staff expected the two results to be the same. There appeared to be little understanding of the concept of repeatability and reproducibility. Second, there was an inherent resistance to change in a conservative industry. Comments were made such as "The existing methods have provided adequate results for our needs for the last 100 years and why should we change?".

The SMRI developed a roadshow presentation to present to senior management and laboratory staff to explain and highlight the concepts of analysis, repeatability, reproducibility, the advantages of near infrared transmission spectroscopy and also introduced a SMRI "Toolbox". The factory laboratories currently only analyse process samples on a shift or daily basis using mainly polarimetry and refractometry and are sample constrained due to time and personnel resources. The presentation showed how using the same of number of staff, more analysis could be undertaken on any particular shift resulting in better control. Process trouble-shooting within the factory is limited to the standard analyses that are able to be performed by the factory staff with more onerous testing being left to consultants or SMRI staff, when available. The Toolbox was designed to use the near infrared transmission spectroscopy analyte results (currently unavailable to the factory staff) and produce information that could be used to troubleshoot and improve process control within the factory. Two examples are considered.

Example 1: Centrifuges. Most raw sugar factories use batch centrifuges to separate the sugar crystal from the mother liquor in which the crystal is grown. The basket within the centrifuge is lined with a screen to hold back approximately 1.5 tonnes of sugar as the mother liquor is spun off the sugar, through specific sized apertures in the screen. Occasionally holes or tears can appear in the screen allowing the sugar to escape with the mother liquor resulting in a loss of product. The conventional analysis to check for a torn screen, known as the Target Purity Difference (TPD), takes approximately one day requiring analysis of the centrifuge crystal/mother liquor feed and separated mother liquor. With a bank of 5-7 centrifuges, each machine can only be checked about once per week. When using the near infrared transmission spectroscopy method, both samples are diluted and analysed in less than $15 \mathrm{~min}$. The SMRI Toolbox integrates with the near infrared transmission spectroscopy results (sucrose, brix, ash, glucose and fructose) and calculates the TPD. The time saving allows all centrifuges to be checked every shift. The saving of sugar, based on conservative estimates, is approximately $\$ 750,000$ per factory per year, a saving which goes directly to the financial bottom line. This procedure has already been implemented at a South African factory with the necessary calculations transferred out of the Toolbox and included within their normal Laboratory Information System. Management receive daily reports highlighting each centrifuge's performance on a shift basis.

Example 2: Inversion losses. All sugar factories need to concentrate juices to a concentrated syrup as a first step in sugar isolation; from approximately $14 \%$ to $70 \%$ solids. This is accomplished in evaporator vessels connected in series in a process known as multiple effect evaporation. Temperatures in excess of $112^{\circ} \mathrm{C}$ are used which, depending on solution $\mathrm{pH}$ and time within a vessel, can lead to inversion or sucrose hydrolysis (breakdown of sucrose to glucose and fructose) which is a loss of final product. The conventional procedure for measuring inversion is very expensive using gas chromatography analysis for sucrose, glucose and fructose analysis and has a long lead time (typically two to three weeks per inversion study). The Toolbox provides the means for quick studies as the factory can analyse sucrose, glucose and fructose immediately using the near infrared transmission spectroscopy method. This allows the effect of process changes to be monitored directly. In the last twelve months eight factories have used the Toolbox for inversion studies with one factory undertaking 37 studies in one week. ${ }^{10}$ It has been estimated that the average bottom line saving for the factory is about $\$ 500,000$ per year.

\section{The way forward}

Since the "road show" intervention there has been a renewed interest in using the technology for factory process control and trouble shooting. Three factories are 
currently using near infrared transmission spectroscopy for factory control. In all cases a decision was made not to compare conventional and near infrared transmission results, but rather accept the alternative method results as is and use the increased sample throughput and Toolbox applications as tools to improve production. This has happened in all three cases. A fourth factory will move over to the technology at the start of the new season based solely on environmental issues-the laboratory will no longer need to use lead acetate in some of their analyses removing the need for personnel health monitoring, purchasing of an expensive chemical and having to pay for disposal. A further four factories are currently evaluating the implications on factory operation procedures.

The SMRI is currently developing additional analyte equations that will be added to the SMRI equation sets in the next equation update at the start of the new season. These include additional analytes such as colour and ash (for streams not currently included and a range of refinery streams and raw sugar analysis so that complete week end, month end and annual stock take procedures can be run. Current stocktake procedures use the pol/sucrose ratio which results in incorrect sucrose balances. ${ }^{11}$

\section{References}

1. K.J. Schäffler, A.N. Dunsmore and J.H. Meyer, "Rapid analysis of sugar products by near infra red spectroscopy", Proc. S. Afr. Sug. Technol. Ass. 67, 222-229 (1993).

2. K.J. Schäffler and M.T.D. de Gaye, "Rapid near infrared estimation of multi-components in mixed juice and final molasses: the possibility of day-to-day control of raw sugar factories using NIR", Proc. S. Afr. Sug. Technol. Ass. 71, 153-160 (1997).

3. K.J. Schäffler, "Trials and tribulations of implementing NIRS for raw sugar factory liquors", Proc. S. Afr. Sug. Technol. Ass. 74, 361-368 (2000).

4. K.J. Schäffler, "Automated routine analysis of quality parameters in sugarcane juices and molasses by NIR", Proc. S. Afr. Sug. Technol. Ass. 75, 318-321 (2001).

5. R. Simpson and J. Oxley, "Routine analyses of molasses and mixed juice by NIR spectroscopy", Proc. S. Afr. Sug. Technol. Ass. 81, 245-275 (2008).

6. R. Simpson and Y. Naidoo, "Using near infra-red spectroscopy for rapid quantification of intermediate sugar factory products", Proc. S. Afr. Sug. Technol. Ass. 83, 382-391 (2010).

7. R. Simpson and Y. Naidoo. "Can near infrared spectroscopy (NIRS) be used to predict lactic acid, acetic acid and ethanol in sugarcane mixed juice?", Proc. S. Afr. Sug. Technol. Ass. 84, 432-438 (2011).

8. Y. Naidoo and S. Walford, "The development of juice NIRS predictions for minor constituents", Proc. Int. Soc. Sugar Cane Technol. 28, 8 (2013).

9. S. Walford and S Naidoo, "Light at the end - a season of composite MJ NIRS analysis", Proc. S. Afr. Sug. Technol. Ass. 88, 90-101 (2015).

10. N. Dairam, R. Ramur, S. Ngema, N. Sutar and S. Madho, "Sucrose losses across the Gledhow evaporators determined using NIRS predictions", Proc. S. Afr. Sug. Technol. Ass. 89, 391-405 (2016).

11. B. Barker, S. Madho and S. Rahiman, "Factory control using NIRS: are we there yet?", Proc. S. Afr. Sug. Technol. Ass. 90, 400 (2017). 